\title{
Utility Computing
}

\author{
Ranjan Kumar Mondal ${ }^{1}$ and Debabrata Sarddar $^{2}$ \\ ${ }^{1}$ Department of Computer Science \& Engineering, \\ University of Kalyani, Kalyani, India \\ ${ }^{2}$ Assistant Professor, Department of Computer Science \& Engineering, \\ University of Kalyani, Kalyani, India \\ ${ }^{1}$ ranjan@klyuniv.ac.in, ${ }^{2}$ dsarddar1@gmail.com
}

\begin{abstract}
With the growing demand for computing resources and network capacity, providing scalable and reliable computing service on the Internet becomes a challenging issue. Recently more attention has been paid to the Utility Computing concept aiming to provide computing as a utility service similar to water and electricity. While the concept is very challenging in general we focus our attention in this chapter to a restrictive environment web applications, given the omnipresent use of web applications on the Internet, this environment is rich and important enough to warrant careful research. This paper describes the approaches and challenges in building such a computing platform.
\end{abstract}

Keywords: Utility Computing, Web Applications, Application Servers, Resource Provisioning

\section{Introduction}

Utility computing is technology that enables a service provider to make computing resources and infrastructure management available to customers as needed. This excerpt from Data Lifecycles: Managing Data for Strategic Advantages, discusses how to centralize storage management, improving administration efficiencies and allowing best practices to be applied uniformly across all resources. Read this chapter to learn how you can manage your customers' storage more efficiently, saving them time and money and providing them with important compliance tools.

Utility computing is referred to as a facility provided by providers to users on their demand and also charge them for using service on the basis of specific usage as well as it also provides infrastructure to the consumers. Utility computing is a model of providing facilities on demand of user. The utility model provides the benefit of using the tools maximum on demand as well as better usage of resources and to minimize the costs. "The word utility is an analogy for the customers to use and pay for the quantity of usage. Like electricity is charged on the extent we use rather than paying full part as school fees or college to join class or not but have to pay but the utility computing provides this facility of pay per use."

The term utility computing is getting place in society in different dimensions also like initiative computing, website access, file sharing, different applications, used by the consumers in the markets. Some version of utility computing is chosen from the shared pool utility model which centralizes its computing resources to serve a larger number of users.

Utility computing provides the ability to all customers to access computing services, business processes, and applications from a utility-like service over a network. This influences in term of being saving their money via using the capability on just pay per use. This means all customers can save their money via this utility. 
Utility computing is web computing and storage connected to a managed system and the multiple computing systems that have multiple CPU and networking systems.

\subsection{Definition}

"Utility computing can be simply defined as the "on-demand provisioning of IT resources under the control of the subscriber". It allows users to build, deploy and maintain large applications without the need to invest in owning and operating the physical resources themselves. Instead, the servers, switches, storage and security are owned by a provider and the user is billed only for their usage."[1]

Utility computing is a computing that is based on Pay per Use model. Utility computing offers computational resources on demand service. Cloud computing, grid computing, and managed IT services are based on the concept of utility computing.

Utility computing is the packaging of computing resources, such as computation, storage and services, as a metered service. This model has the advantage of a low or no initial cost to acquire computer resources; instead, computational resources are essentially rented.

Utility computing is a computing that performance a model in which a service provider makes infrastructure management and computing resources such as computation, storage and services. This model has the advantage of a low or no initial cost to acquire computer resources. Like on-demand computing the utility computing searches to maximize the use of resources with minimize costs.

\subsection{Explanation}

Utility computing is a service provider of computing resources and infrastructure management available to customers as required and the specific use rather than as a flat rate, which is a model for the cost of a service that provides them.

Utility computing is one of the most popular IT service models, because of its flexibility and economy it provides. This model is based on that used by conventional utilities such as telephone services, electricity and gas. The principle behind utility computing is simple. The consumer has access to a virtually supply of computing resources over the Internet which can be used whenever it's required. Utility computing solutions can include servers, software, and storage, backup and related solutions. "Cloud computing, grid computing and managed IT services are based on the concept of utility computing."

\section{Utility computing idea}

Utility computing is the computing handling online resources such as data, services that is familiar to a public utility. LPG, water, electricity or telephone are examples. It has the benefit of cheap cost to get computer resources.

The computational resources required to purchase the product has not necessarily turning that is rented. This on demand computing services in a way that became the foundation of the work, cloud computing and software more computing as a service, application and network concept that works reproduction.

\subsection{Concept of Utility Computing}

It is based on that data are stored in an environment which is accessible easily. A user on the network has its own profile so user stores personal data in a central repository rather than on local machines. Applications and services are managed from this common directory. Utility computing is a model in which the software vendor provides a version of it on the Internet. Customers can access the application for use by subscription. 
Utility computing offers clients a cost effective formula without software licenses. It eliminates the costs and risks of installing, supporting and maintaining computer hardware in the enterprise and to maintain necessary staff.

Utility computing opens up new area for software vendors. Software vendors can elaborate their markets, to offer utility solutions to small and medium entrepreneurs.

Utility computing as a Service (UaaS) provides to have potential to impact our daily lives in many ways. Like a company can access to national or international market, including their products without any cost.

\subsection{Examples and Case Studies}

Utility computing is being used in a number of various application areas. Let's consider some examples.

- A Canadian power company is saving about $\$ 500,000$ a year using laptops and PDAs, with some third-party mobile applications, for its 400 field workers. The goal of this mobile computing is to switch from paper trails to fast, accurate digital data paths. The results are savings in paper processing costs and much more accurate maintenance data.

- Men's clothing retailer Ahlers set up a self-service web site through which its retailers can quickly get product information and track orders.

- Russian transportation company Mostransagentstvo built a new system that lets customers make travel reservations immediately.

- Swets Information Services is an outsourcing and facilitating partner for the acquisition, access, and management of scholarly, business, and professional information.

Utility computing can be implemented without cloud computing. Consider a supercomputer that rents out processing time to multiple clients. This is an example of utility computing as users pay for resources used. However, with only one location and no virtualization of resources, it cannot be called cloud computing.

\section{The Basics of Utility Computing}

The principle of utility computing is very simple: One company pays another company for servicing.

The services include software rental, data storage space, use of applications or access to computer processing power. It all depends on what the client wants and what the company can offer.

Many utility computing companies offer packages of resources. A comprehensive package include all of the following: software, including servers, CPUs, monitors, devices, Internet access, including Web servers and browsing software.

Software applications that run the entire range of computer programs, they could include word processing, e-mail clients, applications and everything.

Off-site data storage that is called cloud storage also, there are many reasons a company want to store data off-site. If the company processes a lot of data, it might not have the physical space to hold the data servers it needs. An off-site backup is also a good way to protect information.

Utility computing rates vary depending on the utility computing company and the requested service. Usually, companies charge clients based on service usage rather than a normal charge. The more a client uses services, the more charges it must pay. Some companies bundle services together at a reduced rate, essentially selling computer services in bulk. 


\subsection{History}

In the 1970s and 1980s, mainframe computing comprised huge global computing systems. It was expensive and had a pretty bleak user interface: but, it worked. In the early 1990s enterprises moved to highly distributed client/server computing which allowed IT to deploy PC client systems with, on the face of it, lower cost and a much better end user experience. By the late 1990s, Internet computing allowed systems with the mainframe's centralized deployment and management but with rich PC-like browserbased user experiences.

Now, the industry is in that age of utility computing. Utility computing is a term the IT community has adopted that represents the future strategy of IT. No vendor is embarking alone in this approach - all the major vendors have their own version of this vision. But whatever it is called, utility computing represents an evolution of the way corporations use IT.

\section{Issues of utility computing}

There are several issues that need to be addressed in developing a utility computing system for Web applications:

4.1. Application distribution: The system distributes instances of the application across its network. The system maintains the consistency of different replicas of an application.

4.2. Resource isolation: Given that the platform runs multiple applications on a shared platform that can the applications be isolated from affecting each other. For example, if one application becomes a victim to a denial of service attack, this should not affect other applications.

4.3. Application placement: A variety of metrics can be considered here, including: user proximity, server load, billing, security, relocation cost, customer preferences, DNS caching effect etc.

4.4. Request distribution: When a user sends a request then the approach is to always distribute requests to the closest application servers. However, as we see later consideration of other factors is necessary for the stability of the system. [2]

\section{Characteristics of Utility of Computing:}

The Characteristics of utility computing as follows:

\subsection{Scalability}

The ability to rapidly scale an application can often mean the difference between success and failure for a provider. These resources include CPU, storage, memory and bandwidth, as well as other shared infrastructure. The software itself must be designed to take advantage of increasing resources and the importance of the software architecture cannot be over emphasized. That utility computing provide is the ability to monitor resource utilization.

Utility computing can integrate other technologies, such as virtualization that includes storage virtualization, network virtualization, and workload management.

\subsection{High Availability}

Utility computing provides redundancy at every layer including server as well as platform. Logical servers are controlled by the platform and can be rebuilt by the platform 
in the event of a physical server failure. Physical servers will fail, so the platform must provide the capability to continue operating without affecting the applications.

\subsection{Manageability}

Utility computing provides a platform to deploy any number of servers on a platform with scalable CPU, memory and storage. System administrators can edit, configure, and design applications using web based drag-and-drop editor.

\subsection{Disaster Recovery}

Backup is a struggle for customers that need to ensure their applications and other important data are protected in an ongoing and recoverable manner. The fear of "restore" has been well-founded over the years because attempts to recover applications often resulted in recovery points too far back in time, resulting in data loss.

\subsection{Value}

Most utility providers offer different types of solutions at a fraction of the price of traditional enterprise software solutions. Users can follow these solutions without having any internal IT infrastructure. This requires providers to find ways to incorporate technology solutions that provide the highest levels of efficiency possible. [1]

\section{Architecture Utility Computing}

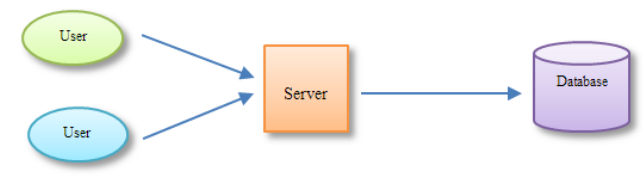

\section{Figure 1. Architecture of Utility Computing}

The utility computing includes servers that are operated by the utility computing platform with the common resources shared different services. The utility computing includes first and some of the second tier servers. The back-end tier includes servers that are outside the scope of the utility computing platform. These typically include back-end database servers and can also include some application servers. Note that back-end database servers themselves could use some sort of dynamic resource sharing. However, this sharing is done using different mechanisms and is outside the scope of this paper. [1].

\section{Function of Utility Computing}

Early personal computers claimed that customers could finish a day's work in a couple of hours using a single device. Some people hoped that computer filing systems and email would create a paperless work environment.

For many companies, the truth about computers is more complicated. It's difficult to predict accurately where problems might occur. On top of that, computer hardware and software evolve rapidly, partly because the computer industry is filled with competitive companies striving to offer the best products and services.

While individual tasks might be easier to complete thanks to computers, the machines themselves can be challenging to maintain and repair. Many companies spend millions of dollars on IT support to keep computers and applications running properly. Even something as simple as adding a new application to a computer network can cause unexpected problems, some corporate executives are looking outside their companies for solutions. One potential approach is to use utility computing. Basically, utility computing 
is a business model in which one company outsources part or all of its computer support to another company.

\section{Cloud vs. Utility Computing}

Utility computing refers to the power to measure the offered services and charge customers for service usage. It is interesting that the term originates from public utility services such as electricity.

Utility computing is connected to cloud computing as it is one of the options for its accounting. As explained utility computing is a good example for less resource demanding applications where top usage is expected to be isolated and uncommon.

"Utility computing does not require Cloud computing and it can be done in any server environment." Also, it is not showing good judgment to measure smaller usage and economically wasteful when applied on a smaller amount. That is often applied on cloud hosting where large resources are being managed.

\section{Benefits}

Utility computing methodology can provide significant cost savings. By delivering IT infrastructure storage as a utility, organizations can:

- Reduce hardware capital expenditures;

- Reduce operating costs;

- Allow IT to align its resources with business initiatives;

- Shorten the time to deploy new or additional resources to users.

\subsection{Advantages of Utility Computing}

1. The client does not need to buy all software and licenses that are needed to use. Instead, the user relies on another party to provide these services. The burden of maintaining and administering the system falls to the utility computing company, allowing the client to concentrate on other tasks.

2. Utility computing gives companies the option to subscribe to a single service and use the same suite of software throughout the entire client organization.

3. Another advantage is compatibility. In a large company with many departments, problems can arise with computing software. Each department might depend on different software suites. The files used by employees in one part of a company might be incompatible with the software used by employees in another part. The most advantage of utility computing is its convenience. The client doesn't have to buy the software and licenses needed to do business. Instead, the client depends on another party to provide these services.

\section{In brief Advantages of Utility Computing}

1. The user requires no software or hardware to buy, just install and maintaining.

2. The software will be paid as they are used.

3. Apart from a PC without web connection, the rest is provided by the utility computing system.

\subsection{Disadvantages of Utility Computing}

Some disadvantages of utility computing are

\section{Potential Disadvantage Is Reliability}

If a utility computing company is in financial trouble or has frequent equipment problems, clients could get cut off from the services for which they're paying. 


\section{Utility Computing Systems Can Also Be Attractive Targets For Hackers}

A hacker might want to access services without paying for them or snoop around and investigate client files. Much of the responsibility of keeping the system safe falls to the provider.

Another disadvantage of utility computing is reliability. If a utility computing company is in any financial trouble or instrumentation problems then users could get off from the services for which they're paying. This is trouble for both the provider and the user. Similarly if a utility computing company goes out of business, its clients could fall in danger. Clients might in problem to hand over tariffs to a smaller company if it could mean losing data and other capabilities should the business suffer.

Utility computing systems can also be attractive targets for hackers and intruder. A hacker may access to services provider without paying and investigate user files. So more responsibility of keeping the system safe falls to the provider but some of it falls on the client's responsibility also

\section{Importance of Utility Computing}

The utility computing always demands a type of cloud scheme this is because it spotlights the business model providing the computing services. The consumer receives resources from utility computing that resources provide service like hardware or software. It is like that you would do for electric service at your home. It is defined in the term of 'pay by the drink'.

\section{Features of Utility Computing}

The one of the advantages and benefits that can be getting from utility computing is better economics. "Corporate data centers are better used in a way that with the resources, often idle 85 percent of the time." The cause of this is the large number of buying the hardware which causes the problem to handle the expected future burdens. So the utility computing plays its best role by allowing the companies to only pay for the resources that they need.

\section{Conclusion}

Utility computing provides a basic platform for resources and a management interface allowing for adjustment of resources based on demand. This type of computing allows providers to focus their services on designing and building a product.

With utility computing on the rise and the need for a highly efficient and flexible model for delivery, the time to consider well-designed utility computing solutions is now. Utility computing providers continue to engage with infrastructure and delivery issues as they search for a reliable mechanism to allow them to remain profitable.

\section{Acknowledgment}

We would like to express our gratitude to Dr. Kalyani Mali, Head of Department, Computer Science and Engineering of University of Kalyani. Without her assistance and guidance, we would not have been able to make use of the university's infrastructure and laboratory facilities for conducting our research.

\section{References}

[1] P. Nickolov, B. Armijo and V. Miloushev, "Globally distributed utility computing cloud", U.S. Patent vol. 429, no. 8, (2013) April 23, pp. 630.

[2] C. Canali, M. Rabinovich and Z. Xiao, "Utility computing for Internet applications", Web Content Delivery, Springer US, (2005), pp. 131-151. 
[3] X. Jiang and D. Xu, "Soda, A service-on-demand architecture for application service hosting utility platforms", High Performance Distributed Computing, 2003, Proceedings 12th IEEE International Symposium on IEEE, (2003).

[4] F. Douglis, A. Haro and M. Rabinovich, "HPP, HTML macro-preprocessing to support dynamic document caching", In Proceedings of the USENIX Symposium on Internet Technologies and Systems, (1997) December, pp. 83-94.

[5] K. Jain and V. V. Vazirani, "Approximation algorithms for metric facility location and k-median problems using the primal-dual schema and lagrangian relaxation", Journal of the ACM, (2001) March.

[6] M. Rabinovich, Z. Xiao and A. Aggarwal, "Computing on the edge: A platform for replicating Internet applications", In Proceedings of the Eighth International Workshop on Web Content Caching and Distribution, (2003) September.

\section{Authors}

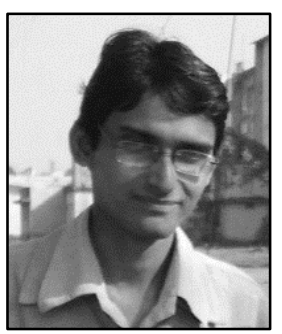

Ranjan Kumar Mondal, received his M.Tech in Computer Science and Engineering from University of Kalyani, Kalyani, Nadia; and B.Tech in Computer Science and Engineering from Government College of Engineering and Textile Technology, Berhampore, Murshidabad, West Bengal under West Bengal University of Technology, West Bengal, India. At present, he is a Ph.D research scholar in Computer Science and Engineering from University of Kalyani. His research interests include Cloud Computing, Wireless and Mobile Communication Systems.

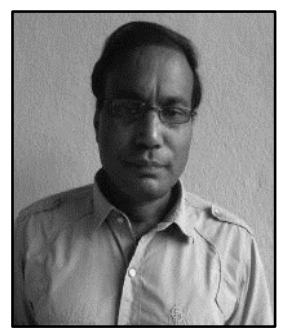

Debabrata Sarddar, is an Assistant Professor at the Department of omputer Science and Engineering, University of Kalyani, Kalyani, Nadia, West Bengal, India. He completed his $\mathrm{PhD}$ from Jadavpur University. He did his M. Tech in Computer Science \& Engineering from DAVV, Indore in 2006, and his B.E in Computer Science \& Engineering from NIT, Durgapur in 2001. He has published more than 75 research papers in different journals and conferences. His research interests include Cloud Computing, Wireless and Mobile Communication Systems. 